\title{
Reduced Osteogenic Differentiation Potential In Vivo in Acute Myeloid Leukaemia Patients Correlates with Decreased BMP4 Expression in Mesenchymal Stromal Cells
}

\author{
Pedro L. Azevedo ${ }^{1}$, Rhayra B. Dias ${ }^{2}$, Liebert P. Nogueira ${ }^{3}$, Simone Maradei ${ }^{4}$, Ricardo Bigni ${ }^{5}$, \\ Jordana S. R. Aragao, Eliana Abdelhay ${ }^{5}$, Renata Binato ${ }^{1}$ \\ ${ }^{1}$ Stem Cell Laboratory, Bone Marrow Transplantation Unit, National Cancer Institute (INCA), Rio de faneiro, Rf, Brazil \\ ${ }^{2}$ Research Division, National Institute of Traumatology and Orthopedics, Rio de Faneiro, Brazil \\ ${ }^{3}$ Oral Research Laboratory, Department of Biomaterials, Institute of Clinical Dentistry, University of Oslo, Oslo, Norway \\ ${ }^{4}$ Bone Marrow Transplantation Unit, National Cancer Institute (INCA), Rio de faneiro, RF, Brazil \\ ${ }^{5}$ Hematology Service, National Cancer Institute (INCA), Rio de faneiro, RF, Brazil
}

The osteogenic differentiation potential of mesenchymal stromal cells (hMSCs) is an essential process for the haematopoiesis and the maintenance of haematopoietic stem cells (HSCs). Therefore, the aim of this work was to evaluate this potential in hMSCs from AML patients (hMSCs-AML) and whether it is associated with BMP4 expression. The results showed that bone formation potential in vivo was reduced in hMSCs-AML compared to hMSCs from healthy donors (hMSCs-HD). Moreover, the fact that hMSCs-AML were not able to develop supportive haematopoietic cells or to differentiate into osteocytes suggests possible changes in the bone marrow microenvironment. Furthermore, the expression of BMP4 was decreased, indicating a lack of gene expression committed to the osteogenic lineage. Overall, these alterations could be associated with changes in the maintenance of HSCs, the leukaemic transformation process and the development of AML.

Keywords: Mesenchymal stromal cells (hMSCs), Acute myeloid leukaemia (AML), Osteogenic differentiation potential, BMP4 gene expression

\section{Introduction}

Although acute myeloid leukaemia (AML) is a heteroge-

Received: August 5, 2021, Revised: October 1, 2021,

Accepted: October 7, 2021, Published online: December 31, 2021

Correspondence to Pedro L. Azevedo

Stem Cell Laboratory, Bone Marrow Transplantation Unit, National Cancer Institute (INCA), Rio de Janeiro, RJ 20230-130, Brazil

Tel: +55-21-3207-1874, Fax: +55-21-2509-2121

E-mail: pedro.azevedo01@hotmail.com

(c) This is an open-access article distributed under the terms of the Creative Commons Attribution Non-Commercial License (http://creativecommons.org/ licenses/by-nc/4.0/), which permits unrestricted non-commercial use, distribution, and reproduction in any medium, provided the original work is properly cited.

Copyright (c) 2022 by the Korean Society for Stem Cell Research neous disease, it is known to have a unique origin from the malignant transformation of normal haematopoietic stem cells (HSCs) into leukaemic stem cells (LSCs) (1). However, what leads to this transformation is still unclear. Several studies suggest that HSCs undergo mutation(s), which gives rise to LSCs. However, not all LSCs present these mutations (2). In this context, changes in signalling in the bone marrow (BM) microenvironment, specifically in mesenchymal stromal cell (hMSC) signalling, could promote malignant transformation (3).

hMSCs play a key role, as they provide essential signals for maintaining and regulating HSCs (3). Various studies, including work from our group, have shown that hMSCs derived from AML patients (hMSCs-AML) are molecularly and functionally altered and that the in vitro osteo- 
genic differentiation potential is decreased (4-6). The ability of hMSCs to differentiate into osteoblasts is essential for the regulation of HSCs, and genes related to osteogenic differentiation are decreased in hMSCs-AML, such as $B M P 4$, which is necessary for maintaining functional HSCs in vivo (7). However, it is not known if decreased BMP4 expression in vitro is associated with alterations in osteogenic differentiation potential and if these findings are reflected in in vivo assays.

In this sense, the aim of this study was to evaluate whether the osteogenic differentiation potential in vivo is reduced in hMSCs-AML and whether it is associated with the expression level of BMP4. For this, we performed a xenotransplantation assay with MSCs-HD and hMSCsAML to induce in vivo bone formation and compared the newly formed tissue, as well as the gene expression of important markers for osteogenic differentiation. With these results, we can improve our knowledge of the mechanisms related to the development of AML.

\section{Materials and Methods}

\section{Patient and healthy donor samples}

BM-derived samples were collected from adult patients with AML at diagnosis (without any treatment) and from adult healthy donors (HDs) registered at the National Cancer Institute (Rio de Janeiro, Brazil). All samples were obtained in accordance with the guidelines of the local Ethics Committee and the Declaration of Helsinki. This study was approved by the INCA Ethics Committee (CAAE 06281419.0.0000.5274), and all participants signed informed consent forms.

\section{Isolation, culture and confirmation of hMSCs}

hMSCs at passage 3 derived from BM samples were isolated and cultured as previously described (4) and were characterized as defined by the International Society for Cellular Therapy (8).

\section{Real-time quantitative PCR (RT-qPCR)}

mRNA was extracted from hMSCs at the end of the process of inducing osteogenic differentiation in vitro, and RT-qPCR was performed as previously described in Azevedo (9). The expression levels of BMP4 were estimated, and B2M and GAPDH were used as normalization genes (Supplementary Table S1).

\section{Subcutaneous xenotransplantation assay}

The subcutaneous xenotransplantation assay with AML-hMSCs and HD-hMSCs was performed as pre- viously reported (10). All animal procedures were performed following the guidelines of the Institutional Animal Care and Use Committee (010/2020).

\section{Implant histology and immunohistochemistry}

The implants obtained were processed for histology and immunohistochemistry analyses as previously reported (10). Rabbit anti-OSX antibody (sc-393325, Santa Cruz Biotechnology, USA) diluted $1: 100$ and goat anti-BMP2/4 antibody (sc-6267, Santa Cruz Biotechnology, USA) diluted $1: 100$ were used.

\section{$\mathrm{X}$-ray microtomography (micro-CT) and morphometric quantification}

MicroCT acquisition was performed as described in Dias (10). The deep learning segmentation tool available on DragonFly (11) was used to separate the newly formed bone from hydroxyapatite. Quantification was performed according to previously described methods (12).

\section{Statistical analysis}

All experiments were carried out in triplicate, and the data are expressed as the mean \pm standard error of the mean. The data were compared using unpaired MannWhitney tests, and a p-value $<0.05$ was considered statistically significant. Statistical analysis was performed, and graphical representations were created using GraphPad Prism $^{\mathrm{TM}}$ software (GraphPad Software Inc.).

\section{Results and Discussion}

All hMSCs used in this study were confirmed by the minimum criteria established by Dominici et al., 2006 (8) (Fig. 1). We observed a reduction in the osteogenic differentiation potential of hMSCs-AML in vitro (Fig. $1 \mathrm{E}$ and $1 \mathrm{~F})$, corroborating other studies $(4,5)$. The findings of conventional in vitro differentiation assays are important and partially predictive of the in vivo physiologic functions of hMSCs, but these cultures do not necessarily reflect the intrinsic physiological potential of the cells. Therefore, to accurately assess the in vivo functional properties of hMSCs in a physiological environment, we used a xenotransplantation assay (13).

Histological examination of the implants revealed that only hMSC-HD cultures formed ectopic ossicles with similar trabecular bone architecture and were able to support haematopoietic stroma (Fig. 2A and 2B). This reconstituted marrow stroma was filled with haematopoietic cells, which is an indicator of the multipotent capacity of hMSCs. 

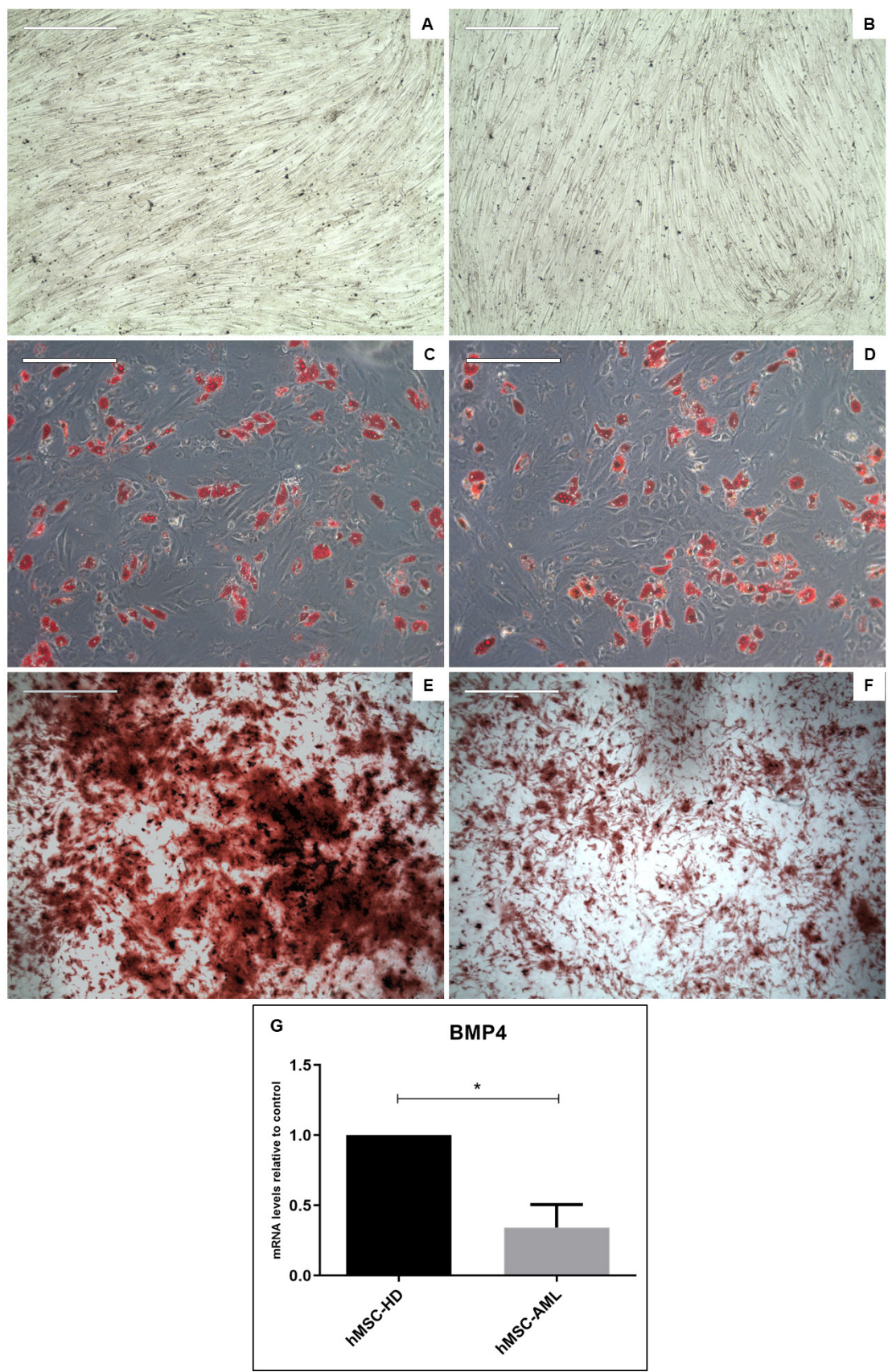

In micro-CT-based 3D reconstruction, bone neoformation was identified in the hMSC-HD (Fig. 2C) and hMSC-AML implants (Fig. 2D); moreover, we observed that the quality (mineral density) of the newly formed tissue was similar under both conditions (Fig. 2H). However, quantitative reductions in the bone volume formed, its thickness, and the relationship between bone volume formed and the volume of the tissue analysed were observed in the implants formed from hMSCs-AML compared to hMSCs-HD (Fig. 2E $\sim$ G). Thus, these results
Fig. 1. hMSC multipotency capacity in vitro. (A, B) Undifferentiated hMSCsHD and hMSCs-AML $(100 \times$ magnification). (C, D) Adipogenic differentiation of hMSCs-HD and hMSCsAML. The accumulation of neutral lipid vacuoles stained with Oil Red O indicates cell differentiation $(20 x$ magnification). (E, F) Osteogenic differentiation of hMSC-HDs and hMSCsAML. Calcium deposition stained with Alizarin Red indicates cell differentiation (20× magnification). (G) BMP4 is downregulated in hMSCs-AML after 21 days of osteogenic induction. To verify BMP4 expression, we used RT-qPCR to determine changes in the mRNA expression obtained from hMSC-AML and hMSC-HD cultures. Data normalization was performed using the endogenous genes $B 2 M$ and $G A P D H$. The bars indicate the mean mRNA expression levels $( \pm$ standard deviation). ${ }^{*} p<0.01$. hMSCs-HD: mesenchymal stromal cells derived from healthy donors; hMSCs-AML: mesenchymal stromal cells derived from AML patients. showed that hMSCs-AML maintained their in vivo bone differentiation capacity, but this capacity was reduced when compared to that of hMSCs-HD.

These results corroborate those of Alice Pievani and colleagues, who observed significant alterations in mature bone formation from hMSCs derived from paediatric AML patients (14), and Frisch and coworkers, where a reduction in mineralized bone tissue formation was described after in vivo assays using an immunocompetent murine model of AML (15). Thus, these changes could be 

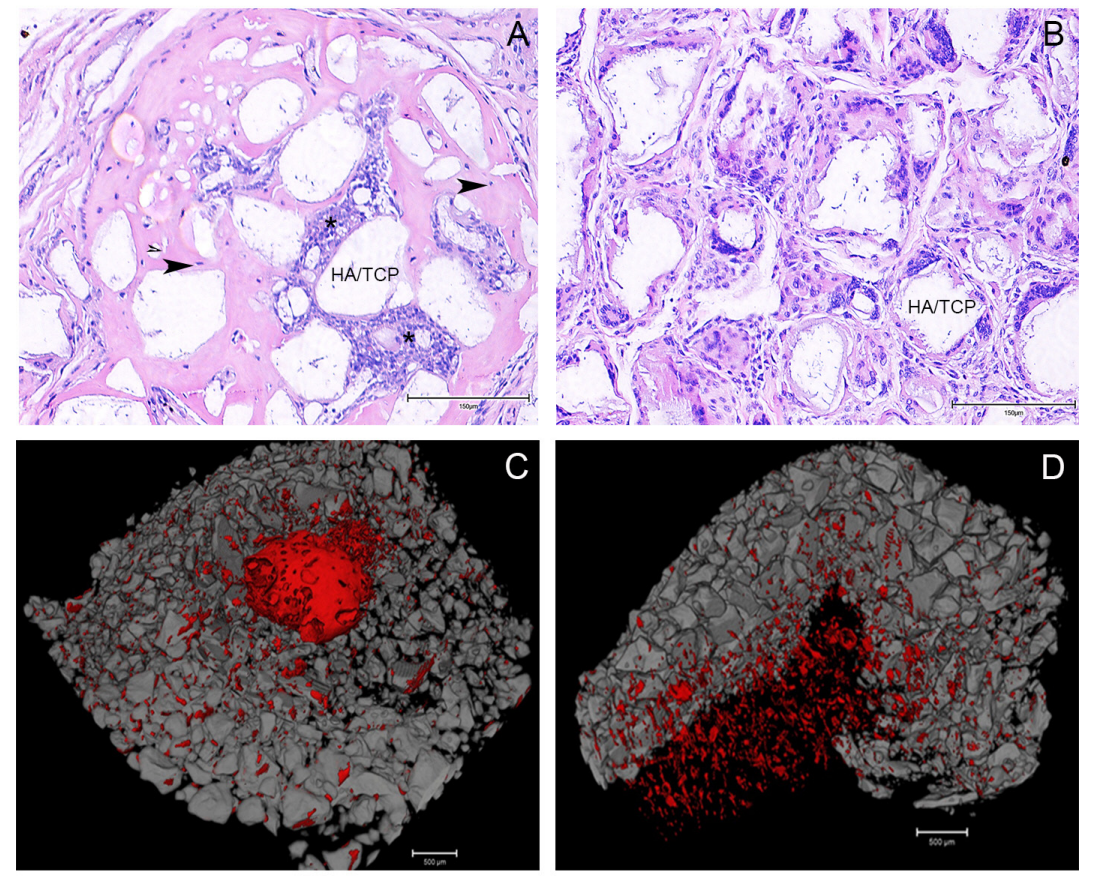
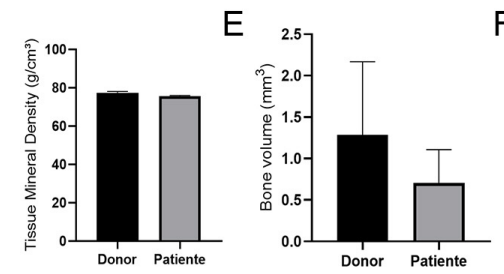

F
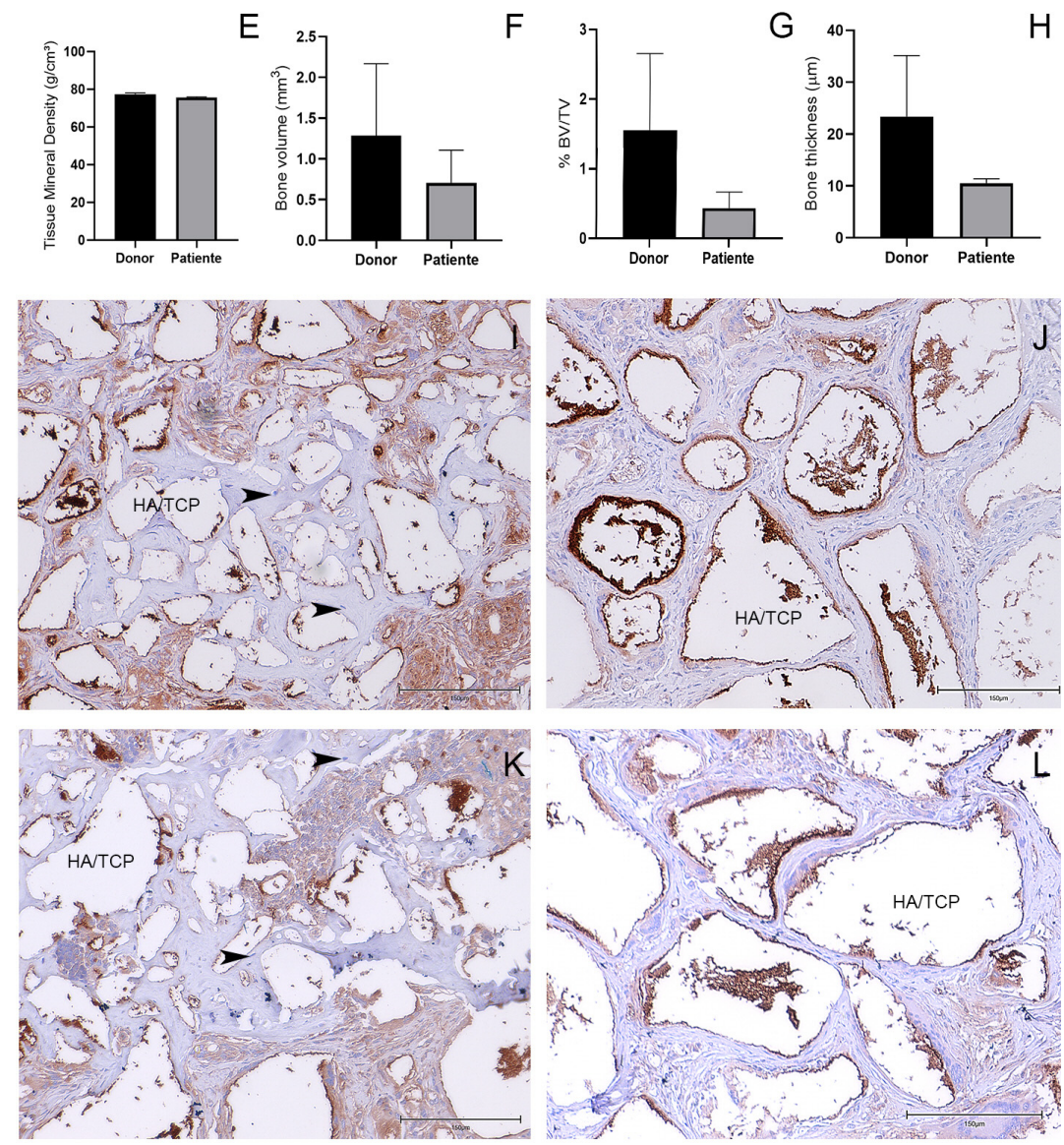

Fig. 2. In vivo osteogenic potential of BMSCs. In vivo transplantation assays were performed by combining hMSCs with HATTCP followed by subcutaneous transplantation into immunocompromised mice. (A, B) H\&E staining. (A) Implants from hMSCHD cultures and (B) hMSC-AML cultures. hMSCs-HD formed ectopic ossicles that were sometimes populated by host haematopoietic marrow (asterisk). The arrowheads indicate osteocytes. $(\mathrm{C} \sim \mathrm{H})$ Micro-CT analysis. (C) Bone tissue formed from hMSCs-HD and (D) hMSCs-AML from the 3D reconstruction of implants. For better visualization of the bone tissue formed (red), part of the HATCP (grey) was removed. $(\mathrm{H})$ Tissue mineral density of implants formed from hMSCsHD and hMSCs-AML. (E) Analysis of bone volume, (F) the relationship between bone volume and tissue volume, $(\mathrm{G})$ and bone tissue thickness in implants formed from hMSCs-HD and hMSCs-AML. (I $\sim \mathrm{L})$ Human origin of the woven bone by immunohistochemical analysis. Expression of BMP4 within the woven bone from (l) hMSCs-HD and (J) hMSCsAML. Expression of Osterix within the woven bone from (K) hMSCs-HD and (L) hMSCs-AML. HATTCP=hydroxyapatite/tricalcium phosphate. associated with the leukaemic transformation process and the development of AML.

This potential of hMSCs to differentiate into osteoblasts in $\mathrm{BM}$ is an essential process for normal haematopoiesis and for the maintenance of HSCs. The process occurs from commitment towards the osteogenic lineage, osteo- 
progenitor cell proliferation, osteoblast maturation and bone matrix mineralization, driven by a complex network of cytokines, hormones, and growth factors (16, 17). Changes in the regulation of this process, associated with lower production of osteoblasts, may result in altered bone deposition and alter the maintenance of HSCs. It is believed that reduced bone deposition can promote the exit of quiescent HSCs from the endosteal niche, associated with an increase in the number of circulating blasts in BM (18).

Interestingly, we also observed that hMSCs-HD were able to develop a supportive haematopoietic stroma and reconstruct an in vivo bone marrow-like microenvironment in mice with haematopoietic cells (Fig. 2A), confirming that hMSCs can organize and are important components of the haematopoietic microenvironment $(13,19)$. This finding was not observed in the implants obtained from hMSCs-AML, evidence that corroborates the report by Priya Chandran and colleagues, who found that hMSCsAML have altered capacity to expand differentiated haematopoietic progenitors in vitro (20).

$B M P 4$, a member of the transforming growth factor $\beta$ $(T G F-\beta)$ superfamily of secreted signalling molecules, was initially identified for its ability to induce bone formation (21) and plays an important role in osteogenic differentiation. It induces the commitment of hMSCs towards the osteogenic lineage and enhances the activity of mature osteoblasts (16). BMP receptor activation in osteogenesis, involves both $S m a d 1 / 5 / 8$ and $M A P K$ downstream signaling activation, and works in conjunction with Osterix via both Runx2 dependent and independent pathways (22). In mice, has already been evidenced that overexpressing BMP4 had enlarged bones containing thick trabeculae (23), and the loss of BMP4 resulted in severe impairment of osteogenesis (24). In this work, the BMP4 expression of hMSCs-AML after 21 days of osteogenic induction in vitro was also decreased (Fig. 1G), similar to undifferentiated hMSCs-AML (4), indicating that it could be associated with reduced osteogenic potential. In fact, it was not possible to observe the expression of $B M P 4$ in implants obtained in vivo from hMSCs-AML (Fig. 2J) when comparing intense marking in the matrix adjacent to new bone formed from the hMSCs-HD (Fig. 2I).

In addition, $B M P 4$ is a critical component produced by haematopoietic microenvironment that regulates both HSCs number and function $(7,25)$. Thus, a decreased of BMP4 expression in implants obtained in vivo from hMSCs-AML, could be associated with the changes in HSCs, contribute to the suppressing normal hematopoiesis, the leukaemic transformation and culminate in the
AML.

Finally, osteoblast differentiation is a multistep process in which hMSCs differentiate into osteoblast lineage cells, including osteocytes. Osterix (OSX) is an osteoblast-specific transcription factor that activates a repertoire of genes during the differentiation of preosteoblasts into mature osteoblasts and osteocytes (16). Similar to BMP4, we observed OSX expression in hMSC-HD implants only (Fig. 2K), confirming that there was alteration in osteogenic differentiation and formation of mature osteoblasts in hMSCs-AML.

In conclusion, the current study showed a reduction in osteogenic differentiation potential in vivo and an absence of osteocytes and haematopoietic support when hMSCsAML were used, in contrast to what we found using hMSCs-HD. These findings correlate with the decreased expression of key osteogenic markers such as BMP4 and OSX. These alterations could influence the BM microenvironment, promoting the release of HSCs from the quiescent niche and indirectly being important factors in the leukaemic transformation process and leukaemia progression.

\section{Acknowledgments}

This work was financially supported by FAPERJ (E-26/202.874/2015) and CNPq (308121/2018-0).

\section{Potential Conflict of Interest}

The authors have no conflicting financial interest.

\section{Author Contributions}

PLA performed all experiments, drafted the manuscript, participated in the study design and contributed intellectual content; RBD performed xenotransplantation, histology and immunohistochemistry assays; LPN performed X-ray microtomography (micro-CT) and morphometric quantification; SM, RBigni and JSRA provided and classified all patient samples used in this study; EA participated in the study design, provided financial support and contributed intellectual content and RB conceived the study and its design and coordination, provided financial support, drafted the manuscript and contributed intellectual content. All authors read and approved the final manuscript.

\section{Supplementary Materials}

Supplementary data including one table can be found with this article online at https://doi.org/10.15283/ijsc21138. 


\section{References}

1. Lapidot T, Sirard C, Vormoor J, Murdoch B, Hoang T, Caceres-Cortes J, Minden M, Paterson B, Caligiuri MA, Dick JE. A cell initiating human acute myeloid leukaemia after transplantation into SCID mice. Nature 1994;367:645648

2. Shlush LI, Mitchell A, Heisler L, Abelson S, Ng SWK, Trotman-Grant A, Medeiros JJF, Rao-Bhatia A, JaciwZurakowsky I, Marke R, McLeod JL, Doedens M, Bader G, Voisin V, Xu C, McPherson JD, Hudson TJ, Wang JCY, Minden MD, Dick JE. Tracing the origins of relapse in acute myeloid leukaemia to stem cells. Nature 2017;547: 104-108

3. Ladikou EE, Sivaloganathan H, Pepper A, Chevassut T. Acute myeloid leukaemia in its niche: the bone marrow microenvironment in acute myeloid leukaemia. Curr Oncol Rep 2020;22:27

4. Binato R, de Almeida Oliveira NC, Du Rocher B, Abdelhay E. The molecular signature of AML mesenchymal stromal cells reveals candidate genes related to the leukemogenic process. Cancer Lett 2015;369:134-143

5. Geyh S, Rodríguez-Paredes $M$, Jäger P, Khandanpour C, Cadeddu RP, Gutekunst J, Wilk CM, Fenk R, Zilkens C, Hermsen D, Germing U, Kobbe G, Lyko F, Haas R, Schroeder T. Functional inhibition of mesenchymal stromal cells in acute myeloid leukemia. Leukemia 2016;30: 683-691

6. Diaz de la Guardia R, Lopez-Millan B, Lavoie JR, Bueno C, Castaño J, Gómez-Casares M, Vives S, Palomo L, Juan M, Delgado J, Blanco ML, Nomdedeu J, Chaparro A, Fuster JL, Anguita E, Rosu-Myles M, Menéndez P. Detailed characterization of mesenchymal stem/stromal cells from a large cohort of AML patients demonstrates a definitive link to treatment outcomes. Stem Cell Reports 2017;8:1573-1586

7. Goldman DC, Bailey AS, Pfaffle DL, Al Masri A, Christian JL, Fleming WH. BMP4 regulates the hematopoietic stem cell niche. Blood 2009;114:4393-4401

8. Dominici M, Le Blanc K, Mueller I, Slaper-Cortenbach I, Marini F, Krause D, Deans R, Keating A, Prockop Dj, Horwitz E. Minimal criteria for defining multipotent mesenchymal stromal cells. The International Society for Cellular Therapy position statement. Cytotherapy 2006;8: 315-317

9. Azevedo PL, Oliveira NCA, Corrêa S, Castelo-Branco MTL, Abdelhay E, Binato R. Canonical WNT signaling pathway is altered in mesenchymal stromal cells from acute myeloid leukemia patients and is implicated in BMP4 downregulation. Transl Oncol 2019;12:614-625

10. Dias RB, Guimarães JAM, Cury MB, Rocha LR, da Costa ES, Nogueira LP, Hochman-Mendez C, Fortuna-Costa A, Silva AKF, Cunha KS, de Souza SAL, Duarte MEL, Sartore RC, Bonfim DC. The manufacture of GMP-grade bone marrow stromal cells with validated in vivo bone-forming potential in an orthopedic clinical center in Brazil. Stem Cells Int 2019;2019:2608482

11. Object Research Systems. Dragonfly 2020 [Internet]. Montreal: Object Research Systems; 2020 [cited 2020 Jul 20]. Available from: http://www.theobjects.com/dragonfly/.

12. Bouxsein ML, Boyd SK, Christiansen BA, Guldberg RE, Jepsen KJ, Müller R. Guidelines for assessment of bone microstructure in rodents using micro-computed tomography. J Bone Miner Res 2010;25:1468-1486

13. Robey PG, Kuznetsov SA, Riminucci M, Bianco P. Bone marrow stromal cell assays: in vitro and in vivo. Methods Mol Biol 2014;1130:279-293

14. Pievani A, Donsante S, Tomasoni C, Corsi A, Dazzi F, Biondi A, Riminucci M, Serafini M. Acute myeloid leukemia shapes the bone marrow stromal niche in vivo. Haematologica 2021;106:865-870

15. Frisch BJ, Ashton JM, Xing L, Becker MW, Jordan CT, Calvi LM. Functional inhibition of osteoblastic cells in an in vivo mouse model of myeloid leukemia. Blood 2012;119: 540-550

16. Thomas S, Jaganathan BG. Signaling network regulating osteogenesis in mesenchymal stem cells. J Cell Commun Signal 2021 doi: 10.1007/s12079-021-00635-1. [Epub ahead of print]

17. Komori T. Regulation of osteoblast differentiation by transcription factors. J Cell Biochem 2006;99:1233-1239

18. Krause DS, Scadden DT. A hostel for the hostile: the bone marrow niche in hematologic neoplasms. Haematologica 2015;100:1376-1387

19. Sacchetti B, Funari A, Michienzi S, Di Cesare S, Piersanti S, Saggio I, Tagliafico E, Ferrari S, Robey PG, Riminucci M, Bianco P. Self-renewing osteoprogenitors in bone marrow sinusoids can organize a hematopoietic microenvironment. Cell 2007;131:324-336

20. Chandran P, Le Y, Li Y, Sabloff M, Mehic J, Rosu-Myles $M$, Allan DS. Mesenchymal stromal cells from patients with acute myeloid leukemia have altered capacity to expand differentiated hematopoietic progenitors. Leuk Res 2015;39:486-493

21. Urist MR. Bone: formation by autoinduction. Science 1965; 150:893-899

22. James AW. Review of signaling pathways governing MSC osteogenic and adipogenic differentiation. Scientifica (Cairo) 2013;2013:684736

23. Okamoto M, Murai J, Yoshikawa H, Tsumaki N. Bone morphogenetic proteins in bone stimulate osteoclasts and osteoblasts during bone development. J Bone Miner Res 2006;21: 1022-1033

24. Bandyopadhyay A, Tsuji K, Cox K, Harfe BD, Rosen V, Tabin CJ. Genetic analysis of the roles of BMP2, BMP4, and BMP7 in limb patterning and skeletogenesis. PLoS Genet 2006;2:e216

25. Zhang J, Li L. BMP signaling and stem cell regulation. Dev Biol 2005;284:1-11 